ORIGINAL ARTICLE

\title{
First Follow up Response Evaluation of Conventional vs Drug Eluting Bead Transarterial Chemo Embolization: A Comparative Study at Tertiary Care Radiology Centre, Rawalpindi
}

Afshan Fayyaz*, Atif Sheraz, Raza Rahim Hyder, Ghulam Abbas

\section{ABSTRACT}

Objective: To compare treatment with drug eluting beads Transarterial Chemoembolization (DEB TACE) vs Conventional Transarterial chemoembolization (CTACE) in hepatocellular carcinoma (HCC) patients at first follow up.

Study Design: Comparative Interventional Study.

Place and Duration of Study: The study was conducted in the department of Radiology CMH Rawalpindi and Armed Forces Institute of Radiology and Imaging (AFIRI), Rawalpindi from July 2015 to May 2019.

Materials and Methods: 254 patients were included in the study. Conventional TACE was done on 176 patients and DEB TACE was done on 78 patients. All patients were called for follow up after 06 weeks. First response evaluation of treated lesions in these patients was seen on triphasic CT liver after 06 weeks of TACE therapy.

Results: On first follow up, out of 176 patients with cTACE, 'Total response' was seen in 90(51.1\%) patients, 'Partial response' in 76 patients (43.1\%), 'No response' in 04(2.27\%) patients.' Progressive disease' was seen in 06(3.4\%) patients.

DEB TACE was done in 78 patients. Out of these, 27 (34.6\%) patients showed 'Total response'. 50(64.1\%) patients showed 'Partial response', 'Progressive disease' was seen in one patient (.02\%).

Conclusion: The findings of this study have shown that C TACE has a better outcome regarding first response evaluation. 'No response' to treatment was not seen in any patient with DEB TACE.

\section{Key Words: Conventional TACE, DEB TACE, First Response Evaluation, Hepatocellular Carcinoma.}

How to cite this: Fayyaz A, Sheraz A, Hyder RR, Abbas G. First Follow up Response Evaluation of Conventional vs Drug Eluting Bead Transarterial Chemo Embolization: A Comparative Study at Tertiary Care Radiology Centre, Rawalpindi. Life and Science. 2021; 2(4): 151154. doi: http://doi.org/10.37185/LnS.1.1.88

This is an Open Access article distributed under the terms of the Creative Commons Attribution License (http://creativecommons.org/licenses/by/4.0), which permits unrestricted use, distribution, and reproduction in any medium, provided the original work is properly cited.

\section{Introduction}

Hepatocellular carcinoma(HCC) is the second leading cause of death from cancer worldwide. In Pakistan, the incidence of HCC is on the rise and according to one study, Hepatitis C virus (HCV) is the most common etiology in up to $58 \%$ patients and hepatitis $B$ virus (HBV) in $25.3 \%$ cases. ${ }^{1}$ Recent large national surveys suggest an HCV prevalence of $4.8 \%$ and $\mathrm{HBV}$

Department of Radiology

Armed Forces Institute of Radiology and Imaging (AFIRI)

National University of Medical Sciences, Rawalpindi

Correspondence:

Dr. Afshan Fayyaz

Professor of Radiology

Armed Forces Institute of Radiology and Imaging (AFIRI)

National University of Medical Sciences, Rawalpindi

E-mail:afshan_fayyaz@hotmail.com

Funding Source: NIL; Conflict of Interest: NIL

Received: Feb 26, 2020; Revised: Sep 21, 2020

Accepted: July 07, 2021

151 prevalence of $2.5 \% .{ }^{1,2}$ The prevalence of HCC in Pakistan is found to be $3.7-16 \%$ of malignant tumors. ${ }^{3}$ Transarterial chemo embolization (TACE) is currently the most popular treatment strategy for patients with unresectablehepato cellular carcinoma since 2004. The aim of this treatment is to deliver the drug as close to the tumor as possible for maximum drug delivery to the site of interest. Many therapy regimes for hepatocellular carcinoma have since been developed. c TACE is a mixture of chemotherapeutic agent (doxurubicin) and a cancer avid agent (lipiodol) which is delivered via catheter to the tumor. This may be followed by embolization with embolic materials such as PVA particles/ embospheres/gelfoam etc. The other regimen is drug eluting beads or DEB. These beads are eluted with chemotherapeutic agent. These beads slowly release the drug over a period of time and embolize the feeding artery at the 
same time. There is lack of evidence regarding efficacy of c TACE vs DEB TACE based on first response evaluation. This study aims to compare the efficacy of c TACE vs DEB TACEin a single speciality institution at tertiary care setting.

\section{Materials and Methods}

All patient with hepatocellular carcinoma with in child Pugh class ' $A$ ' and 'B' who reported for TACE were included in the study. All those patients whose data was incomplete, or those patients who did not report for follow up after 06 weeks were excluded from the study. Method of sampling was convenience non probability. The sample size of 254 was calculated with assumption of 5\% error at $95 \%$ $\mathrm{Cl}$, with an output product (p) of $16 \%$ at $15 \%$ attrition (lost to follow up).

Informed consent of the patient was obtained. All interventions were carried out through groin with femoral punctures on Siemens monoplane floor mounted angiography system Artis Zee. 5/6F sheath was used for all patients. Celiac artery was engaged with 5F Cobra/ SIM I catheter. Micro catheter was used in 207 patients (81\%). Selective catheterization in which catheter was selectively engaged in the vessel supplying the tumor was achieved in 174 patients and non selective catheterization in which the tumor supplying vessel could not be selectively engaged was done in 80 patients.

Patients were divided into 02 groups. In the first group, c TACE was done with inj Doxorubicin $50 \mathrm{mg}$ mixed with lipiodol $10 \mathrm{ml}$, reconstituted as a slurry. In this group there were 176 patients, 97 of whom had single lesion and 79 had multiple lesions. Selective catheterization was achieved in 74 patients with single lesion and 37 patients with multiple lesions. $c$ TACE was performed non selectively in 65 patients. In the second group, drug eluting beads with size of $30-60 \mu \mathrm{m}$ were used. There were 78 patients in this group, 60 patients with single lesions and 18 patients with multiple lesions. Patient segregation as to which drug was to be used was based on number of lesions and the availability and affordability of patient for the particular therapy. Generally for localized lesions, DEB TACE was preferred. For wide spread lesions, c TACE was advised. Options were discussed with the patients. All patients were called for follow up with fresh triphasic CT scan abdomen after 06 weeks of TACE therapy. Computed Tomography was done on Siemens 128 multislice somatom CT scanner. Any enhancement of treated lesions was evaluated by experienced radiologist trained in liver imaging. First response evaluation was done and patients were categorized into the following four categories.

'Total response' when the patients upon reporting for first follow up showed no enhancement of treated lesions (Fig 1a and 1b).

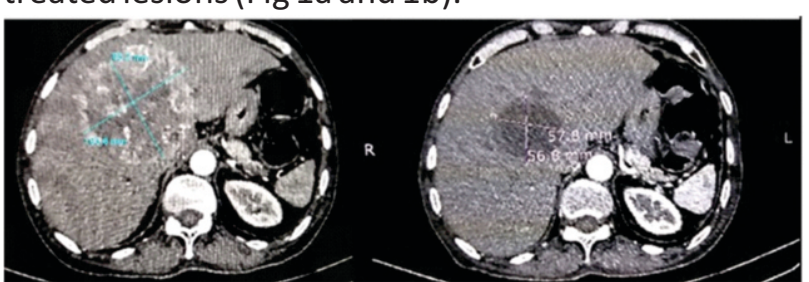

Fig 1a: Pre and post DEB TACE. Note the lesion enhancement pre TACE. Post TACE the same lesion shows no enhancement. There is reduction in size of lesion post TACE

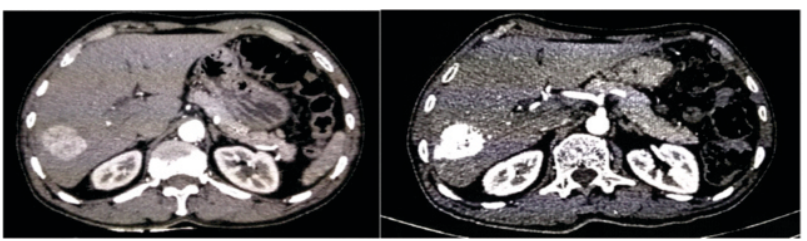

Fig 1b: Pre and post cTACE CT scan of patient, showing good lipiodol retention post TACE

'Partial response' when the lesions showed response with some residual disease (Fig 2).

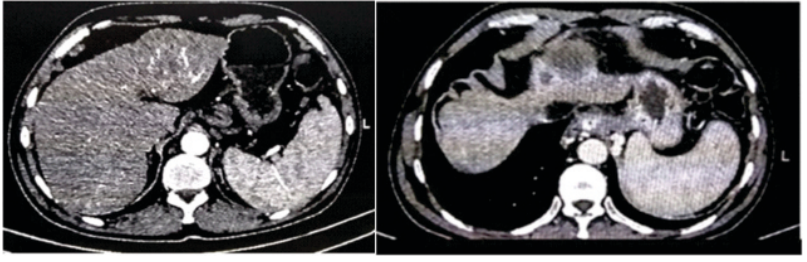

Fig 2: pre (left) and post DEB TACE (right) CT scan of patient with hepatoma. There is enhancement of lesion along right lateral and posterior margin of the treated lesion 'No response' when there was no change between the treated lesion pre and post therapy (Fig 3).
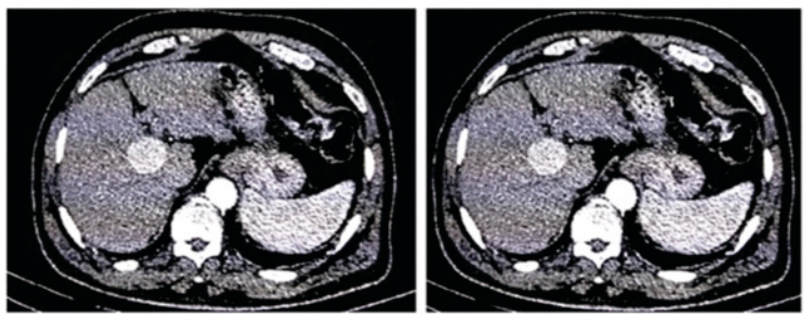

Fig 3: No response to treatment with TACE is observed in this patient treated with c TACE. No lipiodol retention noted. There is avid lesion enhancement pre and post c TACE 
'Progressive disease' when there was interval appearance of new lesions (Fig 4).

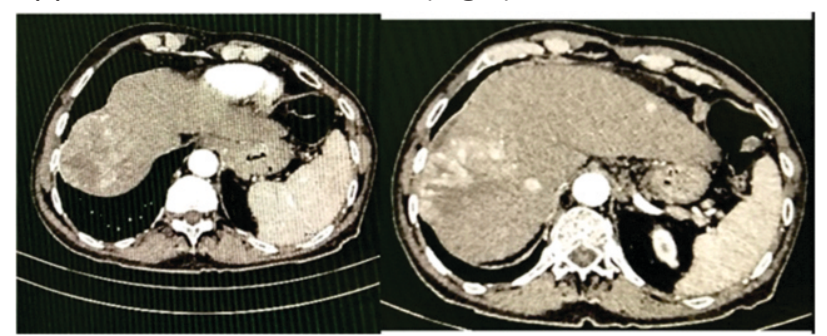

Fig 4: Progressive disease noted in this patient with DEB TACE. There is interval appearance of new lesions 06 weeks post TACE therapy

Data entry was made in SPSS version 21, mean and standard deviation was carried out for frequency variables and chi square test for categorical variables.

\section{Results}

TACE of a total of 460 patients was done over a period from July 2015 to May 2019 in interventional radiology department of Armed Forces institute of Radiology and Imaging. Out of these 460 patients, 254 patients could be followed up. The rest of the patients did not report for follow up. Out of these 254 patients, 192 (75.5\%) patients were males and $62(24.5 \%)$ patients were female patients with Male:Female ratio of 3:1. Age range of the patients

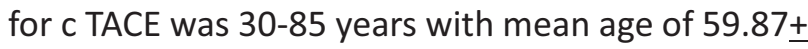
8.96 years. Age range of patients with DEB TACE was 30-77 years with mean age of $59.09 \pm 8.800$ years. The results of cTACE and DEBTACE in terms of response to treatment are presented in table 1.

\begin{tabular}{lcc}
\hline \multicolumn{3}{l}{ Table 1: Results of c TACE and DEB TACE in terms of response } \\
to treatment
\end{tabular}

Out of 176 patients with c TACE, 98 patients had single tumor with mean tumor size of $6.7+5.66728$, and tumors were multiple in 78 patients. Out of 90 patients showing total response to TACE, 53(58.8\%) of them had single tumor and $37(41.1 \%)$ had multiple tumors. Out of 76 patients showing partial response to treatment, 39 patients(51.3\%) had single tumor and $37(48.6 \%)$ patients had multiple tumors. One of the 04 patient showing no response to treatment, one had prominent AV shunting in the tumor. Another patient showing no response to treatment with c TACE had subsequent DEB TACE, and had a good partial response on follow up. Of the patients showing progressive disease, 02 had single tumors and 04 had multiple tumors.

DEB TACE was done in 78 patients. 60(76.9\%) out of these patients had single tumor and 18 patients $(23.1 \%)$ had more than one tumor. Out of the 27 patients showing total response, 20 of them (74.1\%) had single tumor and $07(25.9 \%)$ had multiple tumors.

Out of the $50(64.1 \%)$ patients showing partial response, 39 patients (78\%) had single tumor and $11(22 \%)$ patients had multiple tumors.

\section{Discussion}

In the study conducted, response evaluation of treated lesions at first follow up at 06 weeks was done and long term follow up (more than 06 weeks) was not reported. In previous studies, long term survival benefit of treatment with c TACE vs DEB TACE has been evaluated with some studies showing benefit of DEB TACE over c TACE, while others showing similar response for both drug therapies.

In this study, DEB TACE was done with particle size of 30-60 $\mu \mathrm{m}$. This particle size swells to a size of more than $200 \mu \mathrm{mol} / \mathrm{I}$ upon contact with doxorubicin. ${ }^{4}$ The smaller sized particles have been shown to have more penetrating ability and consequently cause more tumor necrosis. ${ }^{5}$ Larger particles are believed to cause more proximal embolization. All DEB TACE cases in this setting were carried out with particles of this size.

Many studies show a more complete response with DEB TACE than with c TACE. These studies delineate greater long term benefit of DEB TACE vs C TACE in terms of total response ${ }^{6-11}$, while other studies have shown no significant differences in response between the 02 treatment modalities. ${ }^{12,13}$

We found a more complete response with c TACE at the first follow up of our patients. In one of our patients showing no response with c TACE, DEB TACE showed a good partial response. Studies have reported good response to DEB TACE regimen after poor response to treatment with c TACE. ${ }^{14}$ However more studies are needed.

\section{Conclusion}

In our study, c TACE showed a better 'Total response' 
with few patients showing 'No response'. DEB TACE patients showed some degree of response in all patients. This study provides a baseline frequency of outcome which can provide basis for future cohort studies.

\section{Limitations of study:}

1. There was an asymmetric number of patients for c TACE vs DEB TACE.

2. Lab variables as Alpha feto protein levels and PCR were not taken into consideration while interpreting the results.

\section{Acknowledgement}

We extend gratitude to Master Uzair Khurshid for his help with maintenance of our patient data.

\section{REFERENCES}

1. Butt AS, Abbas Z, Jafri W. Hepatocellular Carcinoma in Pakistan: Where do We Stand? Hepatitis Monthly. 2012; 12: e6023.

2. Parkash $\mathrm{O}$, Hamid S. Next big threat for Pakistan hepatocellular carcinoma (HCC). Journal of Pakistan Medical Association. 2016; 66: 735-9.

3. Dinca H, Pelage JP, Baylatry MT, Ghegediban SH, Pascale F, Manfait M. Why do small size doxorubicin-eluting microspheres induce more tissue necrosis than larger ones? A comparative study in healthy pig liver (oral communication 2206-2). CIRSE Annual meeting, Lisbon 2012.

4. Prajapati HJ, Xing M, Spivey JR, Hanish SI, El-Rayes BF, Kauh JS, et al. Survival, efficacy, and safety of small versus large doxorubicin drug-eluting beads TACE chemoembolization in patients with unresectable HCC. American Journal of Roentgenology. 2014; 203: W706-14.

5. Lee Y, Jung K, Kim D, Choi J, Kim B, Kim S, et al. Conventional versus drug-eluting beads chemoembolization for hepatocellular carcinoma: Emphasis on the impact of tumor size. Journal of Gastroenterology and Hepatology.
2017; 32: 487-96.

6. Zou JH, Zhang L, Ren ZG, Ye SL. Efficacy and safety of cTACEversusDEB-TACE in patients with hepatocellular carcinoma: a meta-analysis. Journal of Digestive Diseases. 2016; 17: 510-17.

7. Gao S, Yang Z, Zheng Z, Yao J, Deng M, Xie H, et al. Doxorubicin-eluting bead versus conventional TACE for unresectable hepatocellular carcinoma: a meta-analysis. Hepato-gastroenterology. 2013; 60: 813-20.

8. Huang $K$, Zhou Q, Wang R, Cheng D, Ma Y. Doxorubicineluting beads versus conventional transarterial chemoembolization for the treatment of hepatocellular carcinoma. Journal of Gastroenterology and Hepatology. 2014; 29: 920-5.

9. Xie Z, Wang X, Peng Y, Zhu S, Ma L, Xiang B, et al. Systematic review comparing the safety and efficacy of conventional and drug-eluting bead transarterial chemoembolization for inoperable hepatocellular carcinoma. Hepatology Research. 2014; 45: 190-200.

10. Song JE, Kim DY. Conventional vs drug eluting beads transarterial chemo embolization for hepatocellular carcinoma.World J Hepatol. 2017; 9: 808-14.

11. Song MJ, Chun HJ, Kim HY, Yoo SH, Park CH, Bae SH, et al. Comparative study between doxorubicin-eluting beads and conventional transarterial chemoembolization for treatment of hepatocellular carcinoma. Journal of hepatology. 2012; 57: 1244-50.

12. Facciorusso A. Drug-eluting beads transarterial chemoembolization for hepatocellular carcinoma: Current state of the art. World Journal of Gastroenterology. 2018; 24: 161-9.

13. Golfieri R, Giampalma E, Renzulli M, Cioni R, Bargellini I, Bartolozzi $C$, et al. Randomised controlled trial of doxorubicin-eluting beads vs conventional chemoembolisation for hepatocellular carcinoma. British Journal of Cancer. 2014; 111: 255-64.

14. Li H, Wu F, Duan M, Zhang G. Drug eluting bead transarterial chemoembolization (TACE) vs conventional TACE in treating hepatocellular carcinoma patients with multiple conventional TACE history. A comparison of efficacy and safety. Medicine: 2019; 98: e15314. 